\title{
Morphological Anatomy of Leaf and Rhizome in Zingiber officinale Roscoe, with Emphasis on Secretory Structures
}

\author{
Huanfang Liu
}

Key Laboratory of Plant Resources Conservation and Sustainable Utilization, Guangdong Provincial Key Laboratory of Digital Botanical Garden, South China Botanical Garden, Chinese Academy of Sciences, Guangzhou 510650, China

\section{Chelsea D. Specht}

School of Integrative Plant Sciences, Section of Plant Biology and the L.H. Bailey Hortorium, Cornell University, Ithaca, NY 14853

\section{Tong Zhao}

Key Laboratory of Plant Resources Conservation and Sustainable Utilization, South China Botanical Garden, Chinese Academy of Sciences, Guangzhou 510650, China; and College of Life Science, University of Chinese Academy of Sciences, Beijing 100049, China

\section{Jingping Liao}

Key Laboratory of Plant Resources Conservation and Sustainable Utilization, South China Botanical Garden, Chinese Academy of Sciences, Guangzhou 510650, China

Additional index words. leaf, morphological anatomy, oil cavities, oil cells, rhizome, Zingiber officinale Roscoe

\begin{abstract}
The morphological anatomy of leaf and rhizome was studied at different developmental stages in Zingiber officinale Roscoe using both light and electron microscopy, with an emphasis on characterizing secretory structures. The results show that the leaf comprises epidermal cells, mesophyll cells, and vascular bundles. Oil and crystal cells are scattered throughout the parenchyma, and some are within or in close contact to the vascular bundle sheath. The rhizome consists of epidermis, cortex, and stele. The pericycle of the rhizome remains meristematic and produces tissues centripetally, whereas the endodermis has no meristematic activity. Starch grains vary in shape from round to oval and vary in size from small to large throughout rhizome development. Oil cells and cavities are scattered and cavities are of lysigenous origin. When mature, the starch grains decrease in abundance while an increasing number of oil cells and cavities are formed. This anatomic characterization provides a theory foundation for medicinal exploitation and utilization of $Z$. officinale Roscoe.
\end{abstract}

Zingiber officinale Roscoe (culinary ginger) is a monocotyledonous rhizomatous plant belonging to the family Zingiberaceae. As an important commercial species, it has been used in China and India as a source of spice and medicine since ancient times (Purseglove et al., 1981), and is now cultivated

\footnotetext{
Received for publication 18 Sept. 2019. Accepted for publication 12 Nov. 2019.

Published online 17 January 2020.

This study was supported by the National Natural Science Foundation of China (31670336, 31200246, 3127039, 31570180) and Key Laboratory of Plant Resources Conservation and Sustainable Utilization, South China Botanical Garden, Chinese Academy of Sciences (Y821171001). We thank Haoran Ding for making some slices.

H.L. is the corresponding author. E-mail: hfliu@ scbg.ac.cn.

This is an open access article distributed under the CC BY-NC-ND license (https://creativecommons. org/licenses/by-nc-nd/4.0/).
}

and used worldwide. The rhizome contains the flavor and pungency of the culinary spice together with the essential volatile oils (Zarate and Yeoman, 1994). Ginger leaves are also aromatic and have been used for flavoring and as traditional medicine (Chan et al., 2011).

Although most research on $Z$. officinale is focused on pharmacological properties and the biochemistry of secondary metabolites (Pino et al., 2004; Singh et al., 2008), comparative root anatomy was systematically examined across the Zingiberaceae, including Z. officinale (Uma and Muthukumar, 2014), and the vascular pattern of the rhizome was characterized in a histological study focused on the rhizome $Z$. officinale (Remashree et al., 1997). In addition, secretory structures were characterized, along with the histochemistry for 14 species of Zingiberaceae including Z. officinale (Indriyani, 2017). This study reported that the rhizome of Zingiberaceae plants contained internal secretory structures located in the pith, and noted the presence of amylum grains, protein granules, and oil droplets in all rhizomes examined with variable densities depending on the species and variety (Indriyani, 2017). However, a limited amount of photographic data were provided for further interpretation.

Although these studies provide information about the presence of various secretory cells and products in mature tissues, they do not consider the developmental morphology and anatomy of the various plant tissues that would provide a temporal context for the production of medicinally important compounds. In our previous research, we demonstrated the importance of developmental data in characterizing the ultrastructure of rhizome secretory cavities in $Z$. officinale (Mu et al., 2015). The aim of this study was to elucidate the anatomic morphology of young and mature leaves and rhizomes in $Z$. officinale, and to use developmental data to help characterize the type and distribution of secretory structures in each organ.

\section{Material and Methods}

Plant material and growth conditions. Z. officinale Roscoe were cultivated in the field in Mar. 2015 in South China Botanical Garden, Chinese Academy of Sciences, Guangzhou, China. The climate is humid and soil type is black. Rhizomes and leaves were collected in May and September, and the samples were cut into small pieces and processed immediately according to the following requirements.

Observation by light microscopy. Samples selected for light microscopy were fixed in formalin-acetic acid-alcohol, and subsequently infiltrated and embedded in paraffin (Berlyn and Miksche, 1976). Serial sections ( $8 \mu \mathrm{m}$ thick) were cut on a Leica (Wetzlar, Germany) RM 2016 rotary microtome and stained with hematoxylin (Johansen, 1940). Sections were observed and photographed with Leica DM5500 light microscope fitted with Leica DFC550 digital camera.

Observation by transmission electron microscopy. Samples selected for transmission electron microscopy (TEM) were fixed overnight in $2 \%$ glutaraldehyde in $0.1 \mathrm{M}$ phosphate buffer, $\mathrm{pH}$ 7.0. Air was removed from the samples under vacuum. Following fixation, the samples were washed in phosphate buffer, fixed in $1 \%$ aqueous osmium tetroxide for 2 hours at room temperature, washed again in buffer, and dehydrated in an ethanol series. Plant specimens were embedded in Epon 812 resin. Sections $(2 \mu \mathrm{m})$ were cut using a LKB-11800 rotary microtome, stained with toluidine blue, and observed and photographed with the OlympusAX70 light microscope fitted with an OlympusDP50 digital camera (Olympus Corporation, Tokyo, Japan). Ultra-thin sections $(80 \mathrm{~nm})$ for TEM were cut using a Leica-Ultracut S ultramicrotome, stained with uranyl acetate/lead citrate (Reynolds, 1963) and observed with a JEM-1010 transmission electron microscope at $90 \mathrm{KV}$.

Histochemistry observation. The essential oil present in the oil structures was detected 
by Sudan III using hand-cut transverse sections of fresh leaf and rhizome. Sections were observed and photographed with Leica DM5500 light microscope fitted with Leica DFC550 digital camera.

\section{Results}

Morphological anatomy of young and mature leaf. The leaf comprises epidermal cells, mesophyll cells, and vascular bundles. In transverse section, the epidermis is uniseriate with stomata on adaxial and abaxial surfaces (Fig. 1A). The adaxial cells are rounded and are larger in size than the irregularly shaped abaxial cells. The epidermis possesses a thin cuticle covering the outer cell wall; the epidermal cells located in vein regions are smaller with thicker walls (white arrow) (Fig. 1A). In the young leaf, the mesophyll is arranged dorsiventrally with palisade parenchyma (pp) forming three to five layers oriented adaxially, and spongy parenchyma (sp) forming a single cell layer toward the abaxial surface (Fig. 1A). The palisade cells contain an abundance of chloroplasts. Vascular bundles are surrounded by a parenchymal sheath (vbs) composed of large cells (Fig. 1A and B). Few air canals are observed in spongy and palisade parenchyma, most of which appear to be connected directly with the guard cells (black arrows) (Fig. 1A and B). In the mature leaf, the spongy parenchyma (sp) expands to become several layers thick, and one or two layers of parenchyma cells appear beneath the adaxial epidermis (Fig. 1C). In the main vein, vascular bundles are distributed regularly below the abaxial epidermis and at alternating intervals with air canals (Fig. 1D). Three to five layers of sclerenchyma cells (sc) are present between the vascular bundles and the abaxial epidermis (Figs. 1E and F). The air canals are surrounded by parenchyma cells with many chloroplasts (ch) located along the cell wall (Fig. 1G), each having many plastoglobuli containing lipoproteins (Figs. 1H and I).

In addition to the vascular bundles described previously, scattered vascular bundles are distributed among the parenchyma cells close to the adaxial epidermis (Fig. 1D, arrows). Xylem vessels degrade at maturity (Fig. 2A) and are surrounded by parenchyma cells with visible endoplasmic reticulum (ER) and mitochondria (M) (Fig. 2B).

Secretory structures in leaf. A few of the epidermal cells are oil cells (oc) or silica cells (si) with thicker walls (Fig. 2C and D). Oil cells are also scattered in the parenchyma cells, and some are located within or in close contact with the vascular bundle sheath (Figs. 1A, B, E, and 2E and F). In addition, many crystal cells (cc) are observed in spongy parenchyma within or in close contact with vascular bundle sheath (Figs. 1B and $2 \mathrm{~F}$ ).

Morphological anatomy of young and mature rhizome. The rhizome consists of an epidermis (ep), cortex (c), and stele (s) (Fig. 3A). The epidermis has leaf scars (lsc)
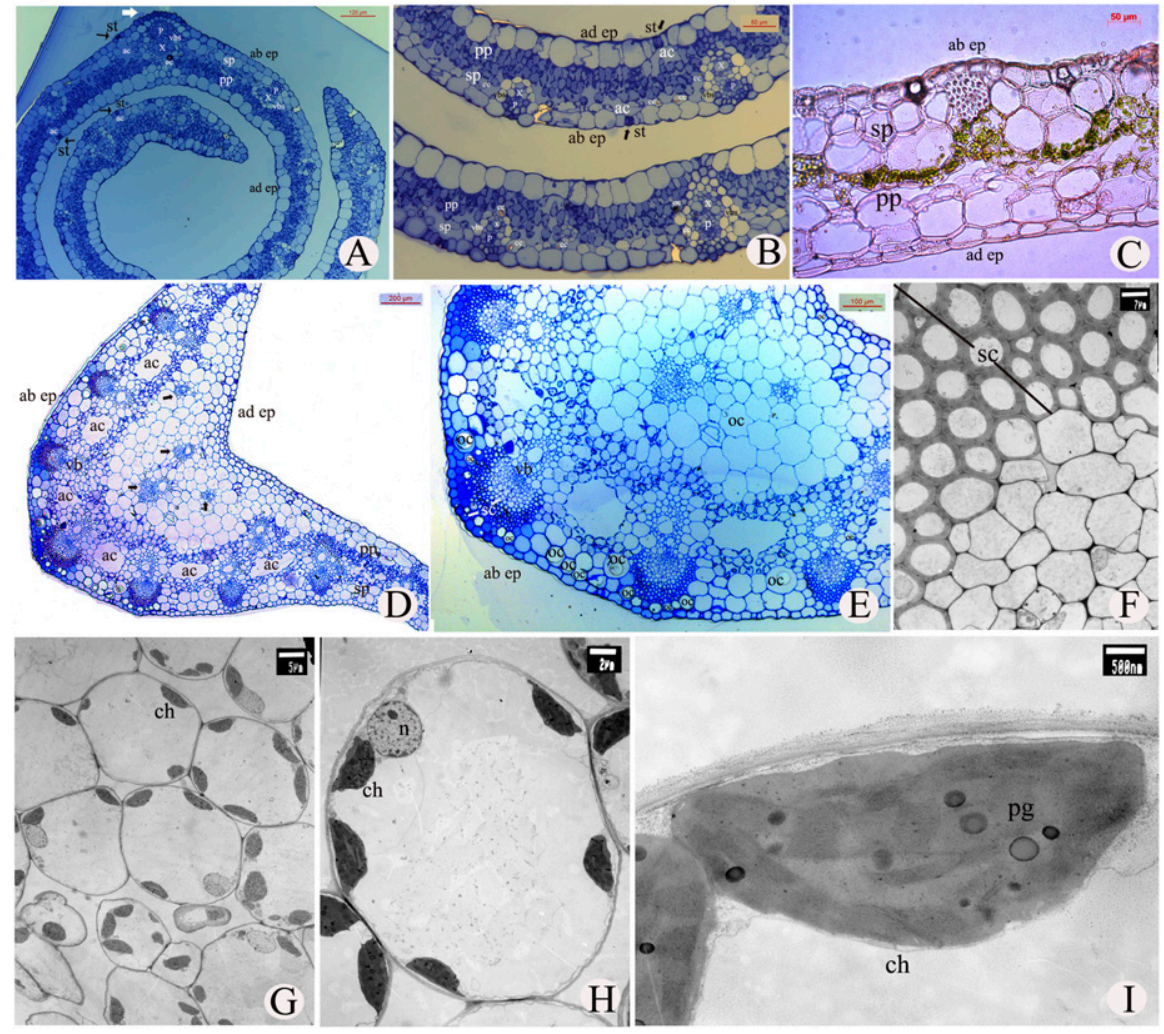

Fig. 1. Anatomy of the leaf. (A) In a young leaf, the epidermal cells located in vein regions are smaller with thicker walls (white arrow), and air canals connected with the guard cells (black arrows). (B) Oil cells and crystal cells scattered in the parenchyma cells. Black arrows indicate guard cells. (C) Mature leaf transverse section. (D) Vascular bundles alternating with air canals in mature leaf. Black arrows indicate scattered vascular bundles. (E) Part of (D) with oil cells scattered in the parenchyma cells and some within or in close contact with the vascular bundle sheath. (F) Sclerenchyma cells. $(\mathbf{G}, \mathbf{H})$ Parenchyma cells with chloroplasts. (I) Chloroplast with plastoglobuli. (A, B, D, E) are semithin sections, (C) is a hand-cut section, and $(\mathbf{F}, \mathbf{G}, \mathbf{H}, \mathbf{I})$ are ultra-thin sections. ab ep = abaxial epidermis; $\mathrm{ac}=$ air canal; ad ep = adaxial epidermis; $\mathrm{cc}=$ crystal cell $; \mathrm{ch}=$ chloroplasts $; \mathrm{n}=$ nucleus; oc $=$ oil cell; $\mathrm{P}=$ phloem $;$ pg = plastoglobuli $; \mathrm{pp}=$ palisade parenchyma; $\mathrm{sc}=$ sclerenchyma cells; $\mathrm{sp}=\mathrm{spongy}$ parenchyma; $\mathrm{st}=$ stomata; $\mathrm{vb}=$ vascular bundle; $\mathrm{vbs}=$ vascular bundle sheath; $\mathrm{X}=\mathrm{xylem}$

and the outer walls of the epidermis are suberized later in development (Fig. 3B and $\mathrm{C}$ ). With development, the cortical cells divide periclinally toward the periphery, forming a multilayered hypodermis (hp) consisting of rectangular thin-walled cells (Fig. 3D and E). The cortex and stele is separated by a single layer of compactly arranged cells, the endodermis (en), which does not exhibit meristematic activity (Fig. 3F). The pericycle (pr) is composed of small and compactly arranged cells situated internally to and contiguous with the endodermis (Fig. 3F). The pericycle appears to induce growth via tangential and radial division (Fig. 3G, arrows). Vascular bundles are also generated from the pericycle (Fig. 3G). With the development, the vascular bundles appear collaterally, forming a ring in the pericycle (Fig. $3 \mathrm{H}$ ). The newly generated cells and vascular bundles enlarge and push toward the center, resulting in continuous primary thickening of the stele while the cortex width remains consistent (Fig. 3A). Later, many scattered vascular bundles are distributed in the cortex and pith, with a higher density in pith than in the cortex (Fig. 3I). Vascular bundles are surrounded by sclerenchymatous cells forming the bundle sheath (vbs) (Fig. 3F). Xylem vessels are highly lignified with scalariform perforation plates (Fig. 4A, arrow), and they degrade with the development of the surrounding parenchyma cells containing organelles including the endoplasmic reticulum (ER) and mitochondria (M) (Fig. 4B).

Cell inclusion and histochemistry of the rhizome. In the young rhizome, few small round starch grains ( $\mathrm{sg}$ ) are observed along the cell wall, with many endoplasmic reticulum (ER) and mitochondria (M) surrounding them (Fig. 4C). With development, the starch grains become oval in shape with plastid inclusions in its corner (Fig. 4D, arrows). Later, abundant starch grains long and oval in shape are formed and located within cells with visible nuclei (Fig. 4E). The cortex and wide pith largely consist of parenchyma cells containing many starch grains, especially in proximity to the endodermis and pericycle (Fig. 3F and $\mathrm{H}$ ). At this stage in development, additional oil droplets appear, each surrounded by endoplasmic reticula (ER) and mitochondria (M) (Fig. 4F). The oil droplets increase in size, and oil cells become increasingly numerous with 


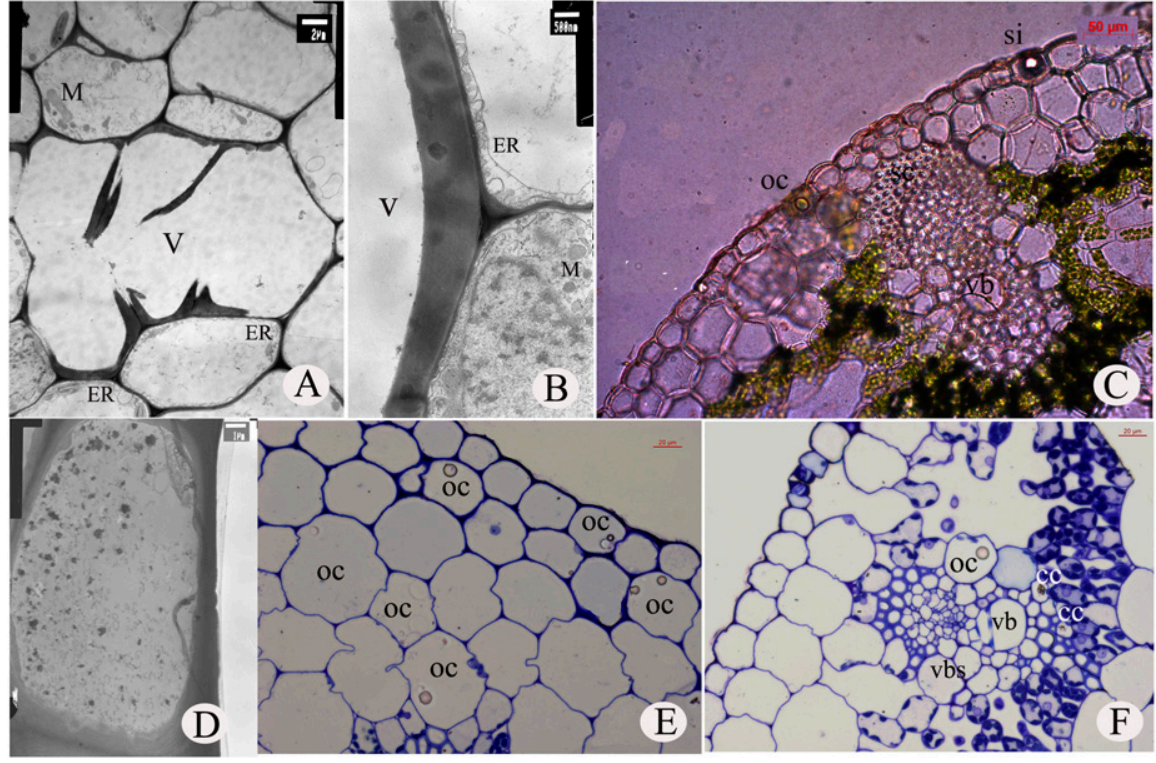

Fig. 2. Anatomy of the leaf. (A) Xylem vessels. (B) Degraded vessel surrounded by parenchyma cells with endoplasmic reticulum and mitochondria. (C) Mature leaf transverse section with oil cell and silica cell in epidermis. (D) An epidermal cell. (E) Oil cells in epidermis and parenchyma cells. (F) Oil cells and crystal cells within or in close contact with the vascular bundle sheath. $(\mathbf{A}, \mathbf{B}, \mathbf{D})$ are ultra-thin sections, (C) is a hand-cut section, and $(\mathbf{E}, \mathbf{F})$ are semithin sections. $\mathrm{cc}=$ crystal cell; ER = endoplasmic reticulum; $\mathrm{M}=$ mitochondrion; $\mathrm{oc}=$ oil cell; $\mathrm{si}=$ silica cell; $\mathrm{V}=$ vessel; $\mathrm{vb}=$ vascular bundle; vbs $=$ vascular bundle sheath
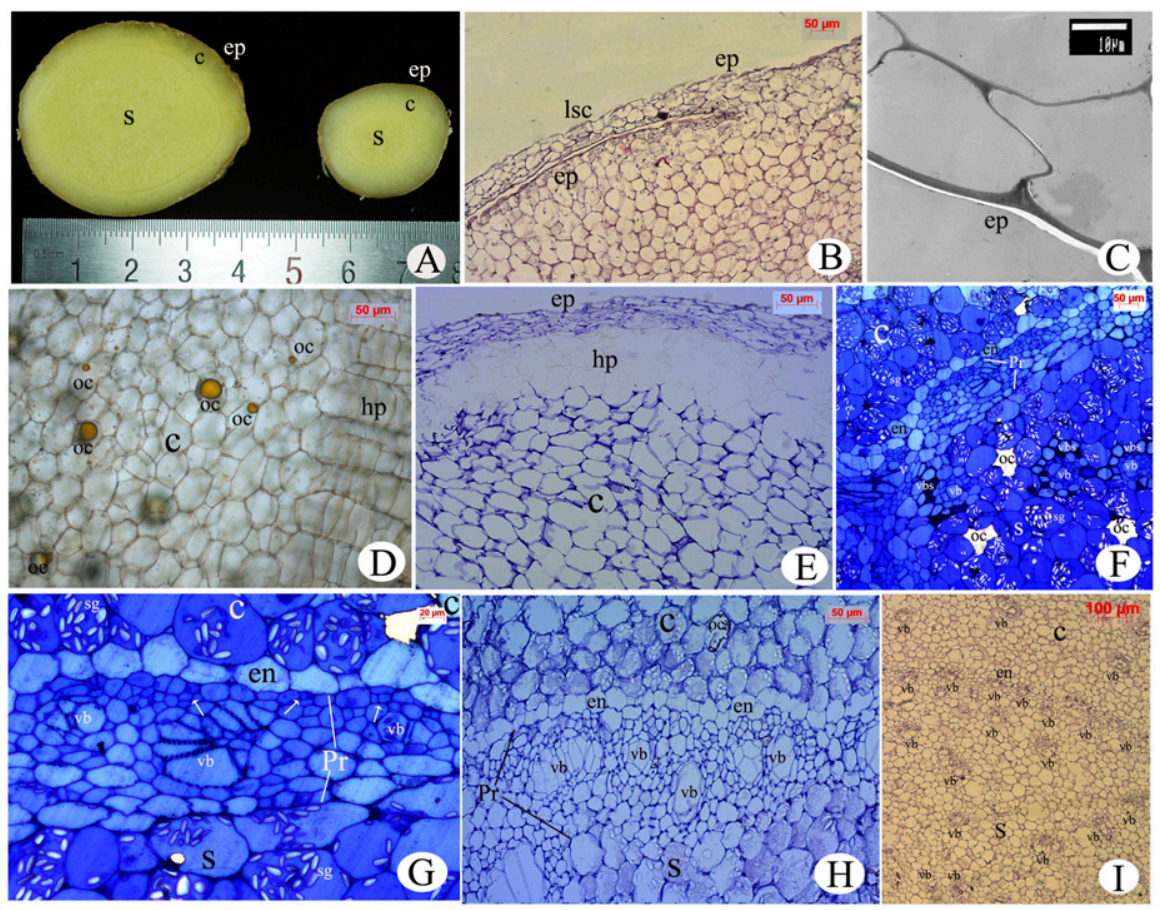

Fig. 3. Anatomy of the rhizome. (A) Transverse section of mature and young rhizome, consisting of epidermis, cortex, and stele. (B) Epidermis with leaf scar. (C) Suberized epidermis. (D) Multilayered hypodermis and oil cells present in cortex. (E) Transverse section of mature rhizome. (F) Cortex and stele with many starch grains in them separated by endodermis and pericycle in young rhizome. $(\mathbf{G})$ Tangential and radial division (arrows) in the pericycle with vascular tissues generated from it in young rhizome. (H) Vascular bundles collateral in a ring in the pericycle. (I) Vascular bundles collateral in a ring and scattered more in pith than cortex in mature rhizome. (A, D) are hand-cut sections, $(\mathbf{B}, \mathbf{E}, \mathbf{H}, \mathbf{I})$ are paraffin sections, $(\mathbf{C})$ is an ultra-thin section, and $(\mathbf{F}, \mathbf{G})$ are semithin sections. bs = bundle sheath; c = cortex; en = endodermis; ep = epidermis; $\mathrm{hp}=$ multilayered hypodermis; $1 \mathrm{sc}=$ leaf scar; $\mathrm{oc}=$ oil cell; $\operatorname{Pr}=$ pericycle; $\mathrm{s}=$ stele; $\mathrm{sg}=$ starch grains; $\mathrm{vb}=$ vascular bundle; $\mathrm{vbs}=$ vascular bundle sheath .

development (Figs. 3D and 4G). Oil cells are surrounded by parenchyma cells that contain few organelles (Fig. 4H). When mature,

the number of starch grains decreases greatly, while more oil cells and cavities are formed (Fig. 4I).

\section{Discussion}

The morphological character in Zingiber. Our research supports previous work by de Menezes et al. (2005) that the stem endodermis of $Z$. officinale does not exhibit meristematic activity, and the pericycle adjacent to the endodermis is the layer responsible for generating vascular tissues. We found that the pericycle produces tissues centripetally, while the endodermis has no meristematic activity. This distribution of meristematic activity results in a continuous primary thickening of the stele, while the cortex width remains consistent. We recommend that this character be regarded as one of the bases for the identification of the ginger rhizome. Currently, the trait of vascular bundles collateral in a ring in endodermis is regarded as a basis for the identification of ginger (Chinese Pharmacopoeia Commission, 2015); based on our research, these vascular bundles are not present in the endodermis, but rather in the pericycle.

In young rhizomes, abundant starch grains appear in the cortex and pith, especially near the endodermis and pericycle. With development, the presence of starch grains decreases significantly, while there is an increase in oil cells and a proliferation of secretory cavities. Zhan et al. (2009) analyzed the chemical composition in seed and fresh ginger and indicated that the oleoresin yields of seed ginger were significantly higher than those in fresh ginger, and seed ginger extract also possessed more gingerols than fresh ginger. Zarate and Yeoman (1994) suggested that flavonoids, curcumin derivatives, phenolics (including gingerol), and essential oils all accumulate within the same cell type in the rhizomes of $Z$. officinale. In our previous research, the ginger essential oil was mainly synthesized in the plastid and cytoplasm, and ultimately accumulates in oil cells and cavities (Mu et al., 2015). Based on this, we regard the oil cells and cavities as the sites for production and accumulation. The number of oil cells and cavities increases with age, so our research may provide an anatomic explanation as to why mature rhizomes are more flavorful than younger ones and are preferentially used medicinally.

The types of secretory cells in Zingiber. Secretory cells are divided into types according to their chemical composition (e.g., oil cells, crystal cells, mucus cells, tannin cells, and myrosinase cells) (Hu, 2012). In our research on $Z$. officinale, we find oil cells and crystal cells present in the leaf, and oil cells along with prominent cavities present in the rhizome.

Three types of secretory cavities have been identified by Fahn (1979), differing in the mechanism by which the intercellular space or lumen of the secretory cavity forms: lysigeny, schizogeny, and schizolysigeny. The difference between lysigenous or schizolysigenous cavity formation is of particular interest, as the autolysis of glandular cells accompanied by the release of their secretion products into the enlarging space as they degenerate may be 


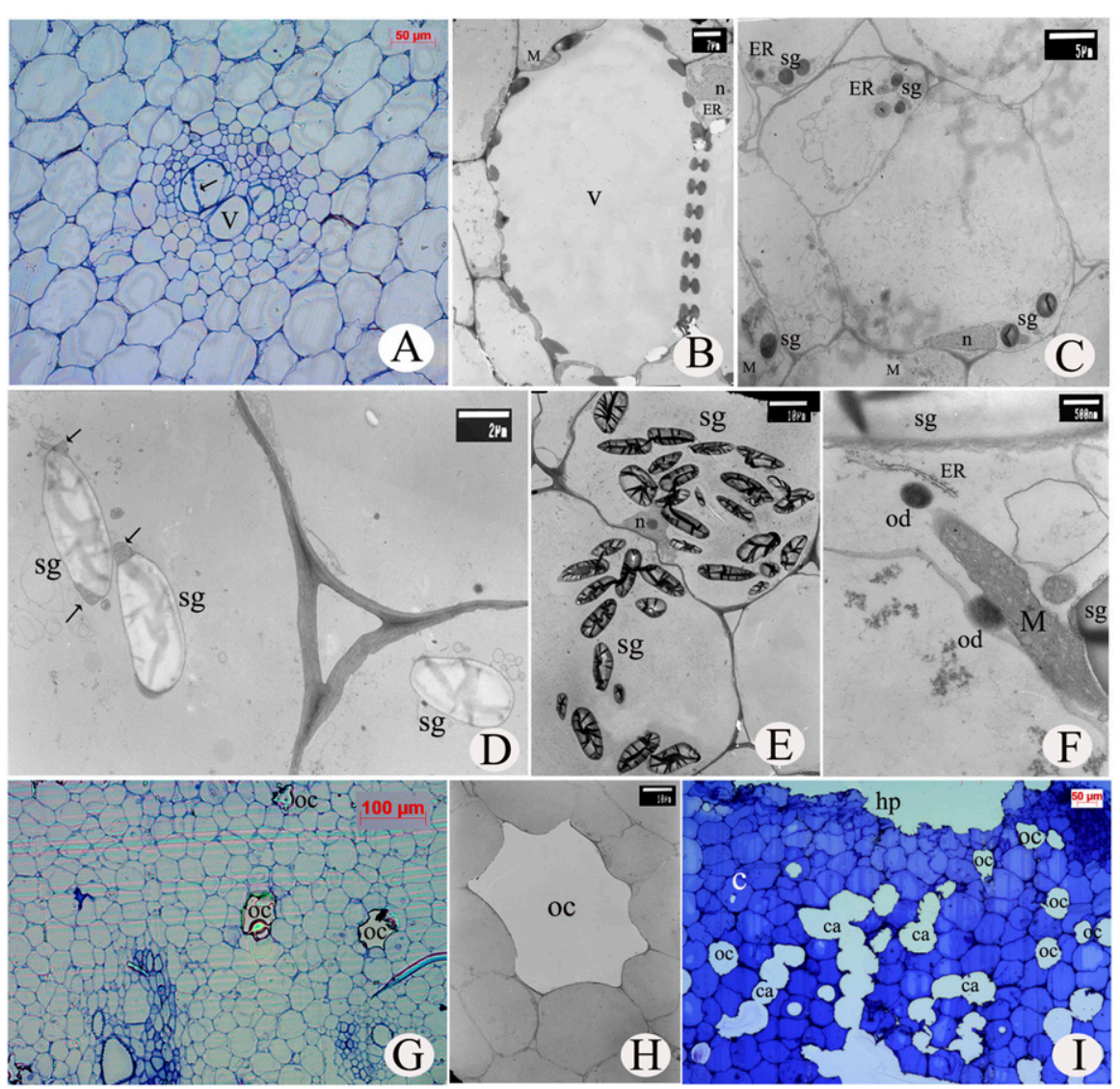

Fig. 4. Anatomy of the rhizome. (A) Xylem vessels with scalariform perforation plates (arrow). (B) Degraded vessel with surrounding parenchyma cells containing endoplasmic reticulum and mitochondria. (C) Round starch grains with endoplasmic reticulum and mitochondria in young rhizome. (D) Oval starch grains with plastid inclusions in its corner (arrows). (E) Abundant starch grains in long and oval shape. (F) Oil droplets with endoplasmic reticula and mitochondria around them. (G) Oil cells in rhizome. (H) Oil cell surrounded by parenchyma cells with few organelles. (I) Mature rhizome with oil cells and cavities. (A, G, I) are semithin sections; $(\mathbf{B}, \mathbf{C}, \mathbf{D}, \mathbf{E}, \mathbf{F}, \mathbf{H})$ are ultra-thin sections. ER = endoplasmic reticulum; $\mathrm{M}=$ mitochondrion; $\mathrm{n}=$ nucleus; $\mathrm{oc}=$ oil cell; od = oil droplet; $\mathrm{sg}=$ starch grain $; \mathrm{V}=$ vessel; $\mathrm{hp}=$ multilayered hypodermis; ca $=$ oil cavity.

considered a true programmed cell death phenomenon (Buchanan et al., 2000). Combined with our previous research on the origin and development of the cavities, in which they were reported to be formed by the disintegration of the oil cells $(\mathrm{Mu}$ et al., 2015), we suggest the oil cavities are of lysigenous origin. Remashree et al. (1997) also reported they were formed Univ. Press, Ames, IA.
Chan, E.W.C., Y.Y. Lim, and S.K. Wong. 2011. Antioxidant properties of ginger leaves: An overview. Free Radic. Res. 1:6-16.

Chinese Pharmacopoeia Commission. 2015. Pharmacopoeia of the People's Republic of China. China Medical Sci. Press, Beijing.

de Menezes, N.L., D.C. Silva, R.C. Arruda, G.F. Melo-de-Pinna, V.A. Cardoso, N.M. Castro, V.L. Scatena, and E. Scremin-dias. 2005. Meristematic activity of the endodermis and the pericycle in the primary thickening in monocotyledons: Considerations on the "PTM". Ann. Brazilian Acad. Sci. 77:259-274.

Indriyani, S. 2017. Secretory structure and histochemistry test of some Zingiberaceae plants. AIP Conference Proc. 1908:040008, https:// doi.org/10.1063/1.5012722.

Fahn, A. 1979. Secretory tissues in plants. Academic Press, London.

Hu, Z.H. 2012. Plant secretory structure anatomy. Shanghai Sci. and Tech. Press, Shanghai.

Johansen, D.A. 1940. Plant microtechnique. McGraw Hill Co., New York.

Mu, N., H.F. Liu, Y.F. Kuang, P. Zou, and J.P. Liao. 2015. Developmental processes of rhizome and ultrastructure of secretory cavities in Zingiber officinale Roscoe. J. Trop. Subtrop. Bot. 23:151-159.

Pino, J.A., R. Marbot, A. Rosado, and A. Batista. 2004. Chemical composition of the essential oil of Zingiber officinale Roscoe from Cuba. J. Essent. Oil Res. 16(3):186-188.

Purseglove, J.W., E.G. Brown, C.L. Green, and S.R.J. Robbins. 1981. Spices, vol. II. Longman, London and New York.

Remashree, A.B., K.K. Sherlija, K. Unnikrishnan, and P.N. Ravindran. 1997. Histological studies on ginger rhizome (Zingiber officinale Rosc.) Phytomorphology 47:67-75.

Reynolds, E.S. 1963. The use of lead citrate at a high $\mathrm{pH}$ as an electron opaque stain in electron microscopy. J. Cell Biol. 17:208-212.

Singh, G., I.P.S. Kapoor, P. Singh, C.S. Heluani, M.P. Lampasona, and C.A.N. Catalan. 2008. Chemistry, antioxidant and antimicrobial investigations on essential oil and oleoresins of Zingiber officinale. Food Chem. Toxicol. 46:3295-3302.

Uma, E. and T. Muthukumar. 2014. Comparative root morphological anatomy of Zingiberaceae. Syst. Biodivers. 12(2):195-209.

entire cell.

\section{Literature Cited}

Berlyn, G.P. and J.P. Miksche. 1976. Botanical microtechnique and cytochemistry. Iowa State

Buchanan, B.B., W. Gruissem, and L.J. Russel. 2000. Biochemistry and molecular biology of plants. Amer. Soc. Plant Physiol, Rockville, MD.
Zarate, R. and M.M. Yeoman. 1994. Studies of the cellular localization of the phenolic pungent principle of ginger, Zingiber officinale Roscoe. New Phytol. 126:295-300.

Zhan, K.Y., H.Z. Yin, X.Z. Zhang, and K. Xu. 2009. Chemical composition analysis on SEF$\mathrm{CO} 2$ extracts of seed ginger and fresh ginger. Food Sci. 30:33-35. 